\title{
Formation of Helices in Graphene Nanoribbons
}

\section{Under Torsion}

\author{
I. Nikiforov ${ }^{\dagger}$ B. Hourahine, ${ }^{\ddagger}$ Th. Frauenheim, ${ }^{\mathrm{I}}{ }^{\mathrm{a}}$ and T. Dumitrică*, ${ }^{\dagger}$ \\ Department of Mechanical Engineering, University of Minnesota, 111 Church Street SE, \\ Minneapolis, MN 55455, USA, Department of Physics, SUPA, University of Strathclyde, John \\ Anderson Building, 107 Rottenrow, Glasgow G4 ONG, United Kingdom, and \\ BCCMS, Universität Bremen, Am Fallturm 1, D-28359 Bremen, Germany \\ E-mail: dtraian@umn.edu
}

\footnotetext{
${ }^{*}$ To whom correspondence should be addressed

†Department of Mechanical Engineering, University of Minnesota, 111 Church Street SE, Minneapolis, MN 55455, USA

${ }^{7}$ Department of Physics, SUPA, University of Strathclyde, John Anderson Building, 107 Rottenrow, Glasgow G4 0NG, United Kingdom

IBCCMS, Universität Bremen, Am Fallturm 1, D-28359 Bremen, Germany
} 


\begin{abstract}
We use objective boundary conditions and self-consistent charge density functional-based tight-binding to simulate at the atomistic-scale the formation of helices in narrow graphene nanoribbons with armchair edges terminated with fluorine and hydrogen. We interpret the microscopic data using an inextensible, unshearable elastic rod model, which considers both bending and torsional strains. When fitted to the atomistic data, the simple rod model uses closed-form solutions for a cubic equation to predict the strain energy and morphology at a given twist angle, and the crossover point between pure torsion and a helix. Our modeling and simulation bring key insights into the origin of the helical graphene morphologies stored inside carbon nanotubes. They can be useful for designing chiral nanoribbons with tailored properties.
\end{abstract}

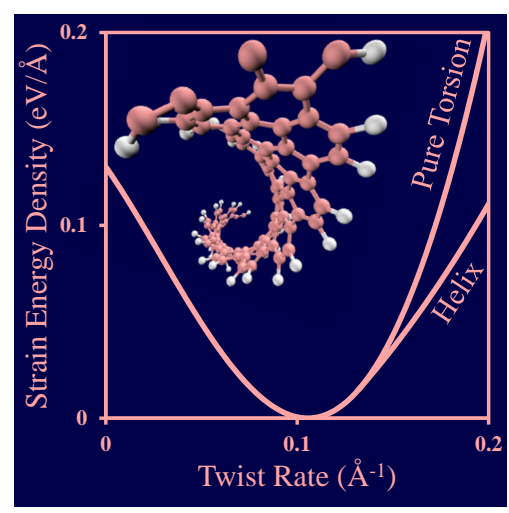

Keywords: graphene nanoribbons, carbon, nanomechanics, rods, SCC-DFTB, objective molecular dynamics

The recent synthesis of graphene nanoribbons (GNRs) encapsulated by carbon nanotubes (CNTs) ${ }^{1-3}$ opens new possibilities in controlling the electronic properties of this material. This is because electronic properties of GNRs are sensitive not only to their width ${ }^{4}$ and edge chemistry ${ }^{5}$ but also to the morphology, including twisted ${ }^{6-9}$ and helical. ${ }^{10}$ The GNR conformation inside CNT is thus important but not fully understood. Both twisted and helical morphologies of GNRs with functionalized edges have been reported inside CNTs. ${ }^{1-3}$ While the interaction of GNRs within the CNT 
(a)
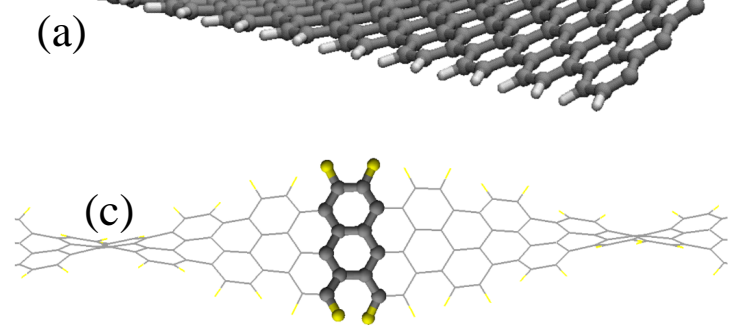

(b)

(d)

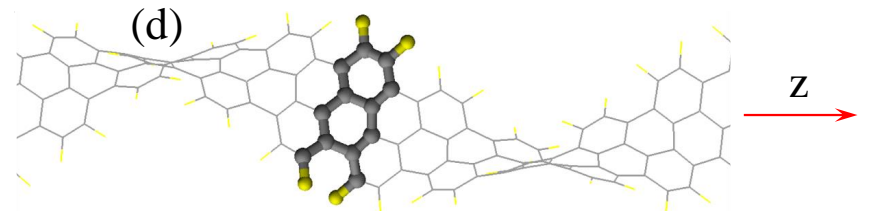

Figure 1: Relaxed configurations of GNRs under torsion. Extended structures of (a) H-terminated, showing aligned edge atoms, and (b) F-terminated, showing staggered edge atoms, GNRs under pure torsion. The ground state of the H-GNR is free of torsion, while the repulsion of the staggered edge atoms causes the F-GNR to have an intrinsic twist (more severe than shown here). For the latter, the Mulliken charges on the edge $\mathrm{F}$ and $\mathrm{C}$ atoms are $-0.19 e$ and $0.26 e$, respectively. Illustrations of helical boundary conditions for simulating GNRs (c) under pure torsion, and (d) in a helical configuration. The simulation cells are shown as ball-and-stick structures, the images are shown as wireframes.

cavity and the formation of twisted GNRs have been explored from different theoretical perspectives, ${ }^{2,10-16}$ the helical equilibria of GNRs has been so far overlooked. It is unclear whether GNRs with decorated edges can be stable helical structures presenting clear preference towards specific helical orientations, or the GNR helicity is induced by the confinement in the CNT cavity.

In this Letter we study the torsional deformation of freestanding GNRs with hydrogen $(\mathrm{H})$ and fluorine $(\mathrm{F})$ armchair edges. The atomic-scale simulations of the helix formation are uniquely enabled by the recent coupling of the objective boundary conditions ${ }^{17}$ with the self-consistent charge density functional-based tight-binding ${ }^{18,19}$ (SCC-DFTB). Boundary conditions for atomistic simulations ${ }^{17}$ based on the concept of objective structures ${ }^{21}$ have already been coupled ${ }^{22}$ with nonorthogonal tight-binding in a manner that allows the application of an arbitrary twist. The method has been used to model a number of tubular ${ }^{23,24}$ and graphene structures ${ }^{24-26}$ including twisted GNRs. ${ }^{7-9,16,27}$ The recent inclusion of the SCC correction ${ }^{18}$ with a helical Ewald sum ${ }^{28}$ improves the description of heteronuclear interactions by considering electron transfer, such as from the $\mathrm{C}$ to the $\mathrm{F}$ edge atoms. ${ }^{20}$ This is important as edge chemistry alone can induce extended deformations in GNRs. ${ }^{7,16}$ We also note that a large amount of simulations is required to understand the helix formation. SCC-DFTB is appropriate for conducting this investigation as its offers both compu- 
tational efficiency and accuracy for geometries, even for biological systems which contain many atomic species. ${ }^{32}$ While empirical constitutive potentials capable of treating C-F interactions exist, including the recent parametrization ${ }^{33}$ of the ReaxFF force field, unfortunately they have not been tested for the present system.

It is intuitive that an object with a large aspect ratio will form a helix when subjected to sufficient torsion, and this is a well-known solution for a Kirchhoff rod under torsion. ${ }^{29-31}$ This helix is a state of combined torsion and bending. The ability of objective boundary conditions to capture these deformations has been already demonstrated in a number of studies, including pure twisting of GNRs ${ }^{8,27}$ and pure bending of an infinite graphene sheet. ${ }^{26}$ Here, we apply objective boundary conditions for the first time the formation of helices with non-zero curvature - a state of combined morphologies with combined bending and torsion. Based on the microscopic data, we show that the formation of the GNR helices can be successfully interpreted with the simple rod model. While the Kirchhoff rod model is widely used in the DNA literature, ${ }^{30}$ its successful application to narrow GNRs is a new development.

Our objective boundary conditions are able to describe both twisted and helical GNR structures as

$$
\begin{gathered}
\mathbf{X}_{i, \zeta}=\zeta \mathbf{T}+\mathbf{R}^{\zeta} \mathbf{X}_{i, 0}, i=1, \ldots, n_{h}, \\
\mathbf{T} \equiv\left[\begin{array}{l}
0 \\
0 \\
T
\end{array}\right], \mathbf{R} \equiv\left[\begin{array}{ccc}
\cos \gamma & -\sin \gamma & 0 \\
\sin \gamma & \cos \gamma & 0 \\
0 & 0 & 1
\end{array}\right]
\end{gathered}
$$

Here, $\mathbf{X}_{i, 0}$ are the coordinates of one of the $n_{h}$ atoms in the objective simulation cell, $\mathbf{X}_{i, \zeta}$ are the coordinates of the $\zeta$-th helical image of this atom, where $-\infty<\zeta<\infty$. T and $\gamma$ are the translation and angle comprising the helical operation. Figure $1(\mathrm{c}, \mathrm{d})$ shows how an infinite GNR under pure torsion (with a straight centerline) or forming a helix (with a helical three dimensional centerline) is built out of a unit cell containing $n_{h}=16$ atoms. The GNRs we consider have six $\mathrm{C}$ dimer lines along their width. 
We carried out conjugate-gradient relaxations of GNRs where the only constraint is the twist angle $\gamma . T$ is allowed to vary freely during the relaxation. This is necessary in order to avoid spurious axial strain, as GNRs exhibit shortening under torsion. ${ }^{8,9,16,27}$ In order to perform direct comparisons between different configurations and with the inextensible rod model, we define the twist rate $\theta=\gamma / T_{0}$ and the strain energy density $W=E / T_{0}$, where $E$ is the strain energy in a single simulation cell. $T_{0}$ is the value the translational component $T$ of the helical operation at the stress-free reference configuration. We carried out two sets of relaxations for each edge type: one set that started with a configuration of pure torsion, and one that started as a helix "cut out" from a CNT. All calculations used 20 uniformly distributed k-points. Relaxations were converged until the maximum atomic force was less than $10^{-6}$ hartrees. At each relaxation step, the selfconsistent charges were considered converged when the maximum charge error was less than $10^{-8}$ elementary charges.

We observed that configurations starting with pure torsion always remained under pure torsion, as this is an equilibrium state even when the helical state is energetically advantageous. The initially helical configurations only remain helical if they are energetically advantageous, otherwise they relax to the pure torsion state. As seen in Figure $2(a, b)$, the stress-free configuration for the H-GNR occurs when at $\gamma=0$ (Figure 1 (a)), and the computed cell length is $T_{0}=4.31 \AA$. For the F-GNR, the stress-free configuration is one of pure torsion (Figure 1 (b)) namely where $\gamma=25.9^{\circ}$. The corresponding length is $T_{0}=4.28 \AA$, meaning the optimal twist rate is $0.105 \AA^{-1}$.

The results of the simulations are summarized in Figure 2, with (a,b) showing the energetics, and (c,d) showing the optimal value of $T$ as a function of $\theta$. The behavior may be summarized as follows: initially, at low values of twist rate, the nanoribbon is centered on the helical axis in a state of pure torsion (Figure 1, (c)). Eventually, the strain energy cost for increasing the torsion becomes too high, and the additional twist rate is accommodated by the GNR assuming a helical configuration that combines torsion and bending strains (Figure 1, (d)). Our simulations show the crossover between pure and helical torsion occurs at twist rates of $\theta \approx 0.063 \AA^{-1}$ and $\theta \approx 0.137 \AA^{-1}$ for the H-GNR and F-GNR, respectively. Note that, even at much higher twist 
rates, the F-GNR has a less severe reduction in $T$ than the H-GNR. This means that the F-GNR forms tighter helices, as $T$ decreases with increasing helix radius $r$. Essentially, because the FGNR possesses intrinsic twist, it prefers a configuration of higher torsion and deviates less from the pure torsion state.
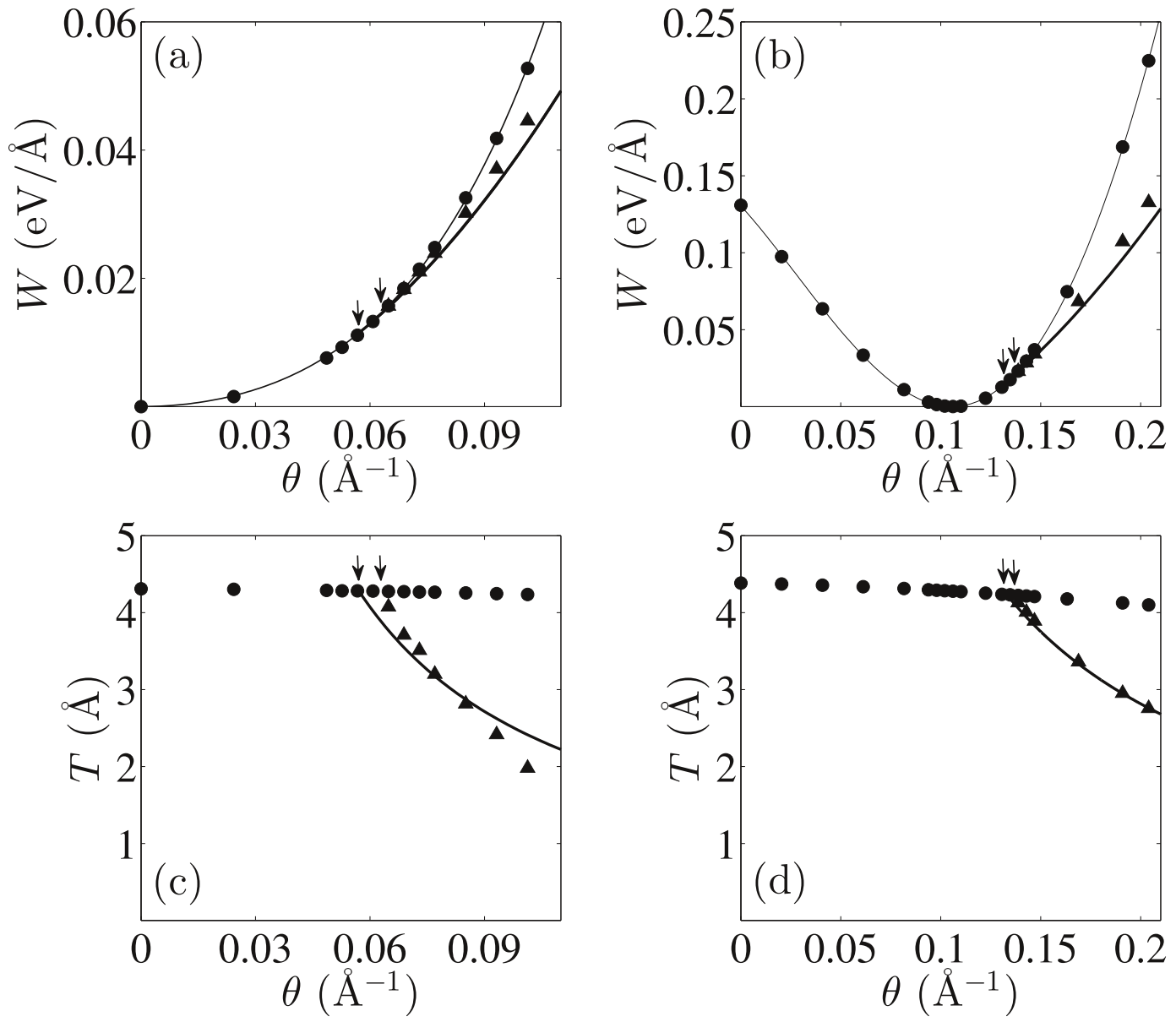

Figure 2: Strain energy density $W$ as a function of twist per undeformed arclength for (a) $\mathrm{H}$ GNR and (b) F-GNR under torsion. Circles indicate simulation results for pure torsion. Thin line indicates fitting of pure torsion with quartic polynomials specified in Table 1. For $\theta$ where a helical equilibrium distinct from pure torsion exists, triangles indicate results of helix simulations and the thick line indicates predictions from the rod model. Arrows indicate crossover points between pure torsion and helical configurations, from simulations and from the rod model. For both edge types, the rod model predicts lower critical twist rate. Translation component $T$ of the helical operation as a function of $\theta$ for (c) H-GNR and (d) F-GNR. Symbols retain their meaning from (a) and (b).

We now attempt to interpret the helix formation using a simple inextensible, unshearable rod model. The uniform helix is a known solution for such a rod with anisotropic bending. ${ }^{30} \mathrm{GNRs}$ are, 
of course, highly anisotropic in bending, as bending within the plane of the ribbon is much more difficult than out of the plane. Because the model rod is inextensible, the arclength of the helix must be equal to $T_{0}$. Thus, given a unit cell length of $T$ and a twist angle $\gamma$, it is straightforward to find the helix radius $r$. Defining $p \equiv \lambda / 2 \pi=T / \gamma$, where $\lambda$ is the pitch of the helix, we have

$$
r=p\left(\frac{T_{0}^{2}}{T^{2}}-1\right)^{1 / 2}
$$

The helix has constant torsion $\tau$ and curvature $\kappa$,

$$
\tau=\frac{p}{\left(r^{2}+p^{2}\right)}, \quad \kappa=\frac{r}{\left(r^{2}+p^{2}\right)}
$$

For anisotropic rods, the material torsion is equal to the geometric torsion. ${ }^{30}$ Then, by assuming that torsional strain and bending strain are superimposable, and as long as the strain energy as a function of pure torsion and pure bending are known, one can minimize the strain energy of the rod model over $T$ at a given $\gamma$ (and therefore $\theta$ ).

The strain energy density, $W_{\tau}$, as a function of torsion is already known from the pure torsion simulations, in which case $\tau=\theta$. Our previous microscopic considerations ${ }^{16}$ demonstrated that this quantity is quartic in torsion,

$$
W_{\tau}=C_{4} \tau^{4}+C_{3} \tau^{3}+C_{2} \tau^{2}+C_{1} \tau+C_{0}
$$

These coefficients were fitted to the pure torsion simulation results. The fitted curves are shown in Figure 2, (a,b), and the coefficients are listed in Table 1. All energies are measured relative to the minimum energy for the respective edge type. Because H-GNRs are achiral (no staggering of the edge atoms), the strain energy is symmetric with respect to the twist sign change (i.e., $\gamma \rightarrow-\gamma$ ). Thus, there are no odd power terms for the H-GNR. The microscopic expressions for the quadratic and quartic coefficients are given in Ref. ${ }^{16}$ Note that the quadratic coefficient depends strongly on $T$. When this coefficient is positive, the intrinsic twist vanishes. 

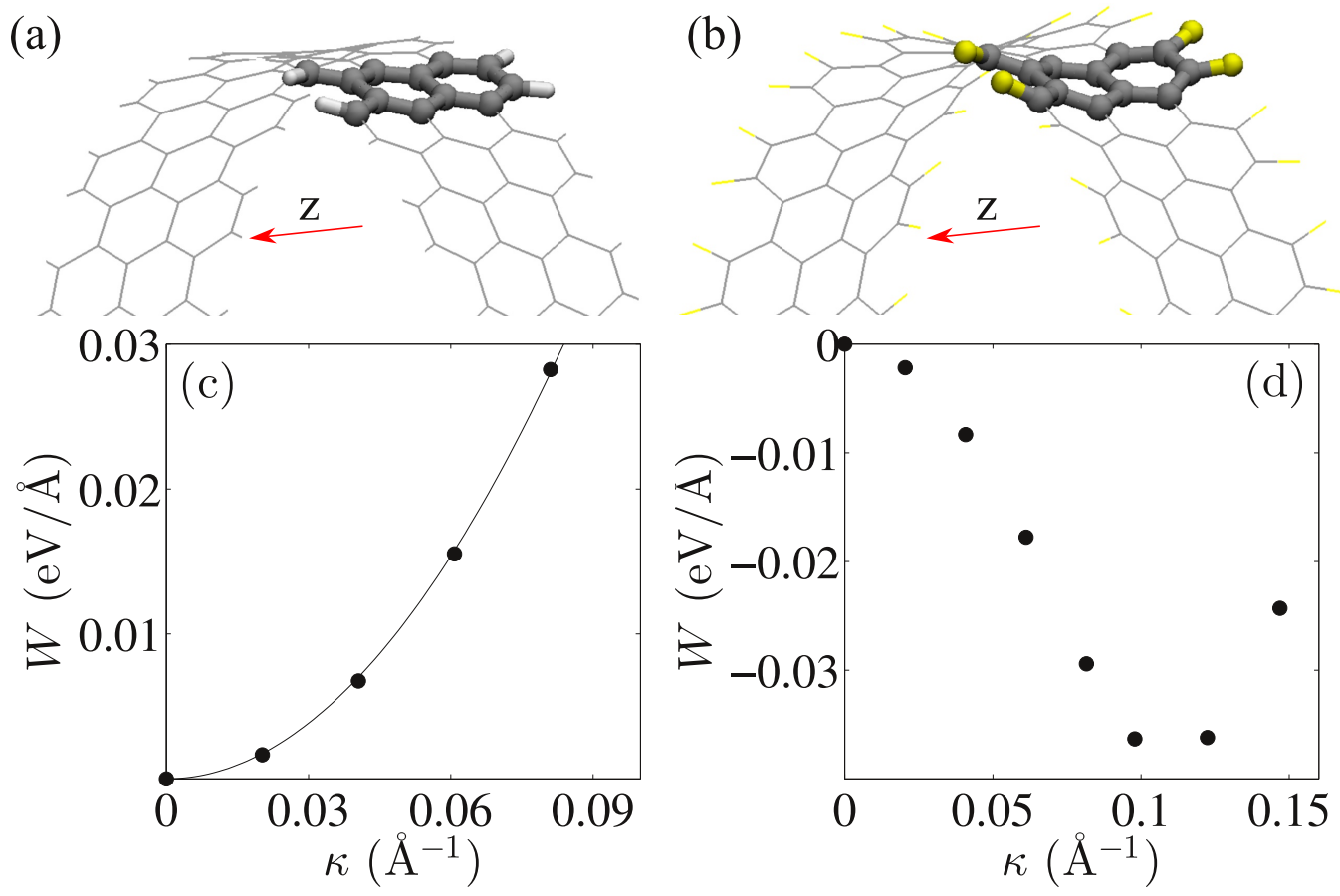

Figure 3: Relaxed configurations of bent (a) H-GNR, showing aligned edge atoms, and (b) FGNR, showing "same-side" edge atoms. The ground state of the H-GNR is at zero curvature, while the repulsion of the edge atoms causes the F-GNR to have an intrinsic curvature (more severe than shown here). The simulation cells are shown as ball-and-stick structures, the images are represented as wireframes. (c) Strain energy $W_{\kappa}$ as a function of curvature $\kappa$ for $\mathrm{H}-\mathrm{GNR}$ under pure bending. Circles indicate simulation results, line indicates fitting. (d) $W_{\kappa}$ as a function of curvature $\kappa$ for F-GNR under pure bending, measured w.r.t. flat configuration free of bending or torsion. Note the intrinsic curvature for this edge termination. 
Table 1: Polynomial coefficients of $W_{\tau}$ for each edge type.

\begin{tabular}{lccccc}
\hline \hline & $\begin{array}{c}C_{4} \\
\left(\mathrm{eV}^{3}\right)\end{array}$ & $\begin{array}{c}C_{3} \\
\left(\mathrm{eV}^{2}\right)\end{array}$ & $\begin{array}{c}C_{2} \\
(\mathrm{eV} \AA)\end{array}$ & $\begin{array}{c}C_{1} \\
(\mathrm{eV})\end{array}$ & $\begin{array}{c}C_{0} \\
(\mathrm{eV} / \AA)\end{array}$ \\
\hline H-GNR & 235.3 & 0 & 2.757 & 0 & 0 \\
F-GNR & -187.8 & 134.4 & -10.38 & -1.416 & 0.1303 \\
\hline \hline
\end{tabular}

The bending strain energy function is also required. To compute it, we simulate GNRs under pure bending. This can be conveniently done using objective boundary conditions. In Eq. (1), $T$ is set to zero, $\gamma$ is set to $2 \pi / N$, where $N$ is an integer, and $\zeta=0, \ldots, N-1$. This simulates a closed ring of constant radius, see Figure 3, (a). Graphene has been shown to behave in a linear elastic manner up to fairly high curvatures,${ }^{26}$ and we expect GNRs to do the same. Thus, we fit the strain energy density as

$$
W_{\kappa}=\frac{1}{2} D \kappa^{2}
$$

where the curvature $\kappa \equiv \gamma / T_{0}$ and $D$ is the bending stiffness. Figure 3, (c) shows bending of the H-GNR fitted with $D=8.54 \mathrm{eV} \AA$.

There are complications when attempting to apply this procedure to the F-GNR. The stressfree F-GNR is twisted, and it is not possible to simulate a structure under both bending and twist, except for cluster simulations restricted to discrete values of twist. Thus, it is necessary to simulate bending of a twist-free F-GNR (Figure 3, (b)). However, under pure bending the F-GNR edges adopt a "same-side" configuration and their repulsion causes an intrinsic curvature in the F-GNR (Figure 3, (d)). We thus do not use the F-GNR bending data in the rod model. Inspection of the relaxed configurations of helical F-GNRs indicates that the edge atoms remain in the "staggered" configuration. This edge configuration is not biased towards either curvature direction, and thus does not possess intrinsic curvature. We furthermore assume that the type of edge atoms do not affect the bending stiffness significantly and use $D=8.54 \mathrm{eV} \AA$ for the F-GNR rod model as well. The close agreement shown below between simulations and rod model justifies this assumption. 
We previously studied the bending of infinite graphene sheets, ${ }^{26}$ applying an approximate analytical molecular orbital analysis to show that bending of graphene is dominated by torsional misalignment of the $\pi$ orbitals. Accompanying simulations using objective boundary conditions predicted a bending stiffness of $1.49 \mathrm{eV}$. Using this information, we can roughly approximate the bending stiffness of the GNRs. There are 12 carbon atoms per unit cell, each taking up $2.65 \AA^{2}$ of area. However, there are four edge atoms per unit cell that do not fully contribute to the torsional misalignment energy. Thus, the stiffness corresponds to between 8 and 12 atoms worth of area per unit cell. Dividing by $T_{0}$, we obtain a range between 7.35 and $11.02 \mathrm{eV} \AA$. This is consistent with our fitted value of $D=8.54 \mathrm{eV} \AA$. This value is slightly below the middle of the expected range, indicating that the fact that the edge $\mathrm{C}$ atoms are bonded to freely-moving $\mathrm{H}$ edge atoms (as opposed to being bonded to carbon atoms that are part of a rigid graphene network) reduces their bending stiffness contribution by more than $50 \%$.

Armed with the bending and torsional strain energy density functions, we can calculate the optimal geometry and strain energy density of the rod model. Substituting Eq. (2) into ?? 3, then substituting into Eq. (4), followed by computing the total strain energy density $W=W_{\tau}+W_{\kappa}$ results in a fourth-order polynomial in $T$ for each given $\theta$

$$
\begin{gathered}
W=\frac{C_{4} \theta^{4} T^{4}}{T_{0}^{4}}+\frac{C_{3} \theta^{3} T^{3}}{T_{0}^{3}}+ \\
\frac{\left(-D / 2+C_{2}\right) \theta^{2} T^{2}}{T_{0}^{2}}+\frac{C_{1} \theta T}{T_{0}}+C_{0}+\frac{D}{2} \theta^{2} .
\end{gathered}
$$

Only minima located at $T_{0}>T>0$ are physically significant. Such a minimum can be found by differentiating the quartic $W$ function and and solving the resulting cubic. For the structures and ranges of $\theta$ studied here, if an extremum appeared in the range $T_{0}>T>0$, it was always found to be a minimum. The lowest $\theta$ at which such a minimum appears represents the crossover between pure and helical torsion. The rod model predicts these crossovers occurring at $\theta=0.057 \AA^{-1}$ and $\theta=0.132 \AA^{-1}$ for the H-GNR and F-GNR, respectively, in good agreement with the values predicted by simulations at $\theta \approx 0.063 \AA^{-1}$ and $\theta \approx 0.137 \AA^{-1}$. It thus predicts that the stress-free 
configuration must be one of pure torsion (or zero torsion for the H-GNR), as opposed to a helical configuration.

Figure 2(a,b) shows the strain energy densities $W$ predicted by the rod model in the helical state, while (c,d) shows the corresponding values of $T$. Despite the simplicity of the model, the agreement is reasonable, especially in the estimation of the crossover point. That the agreement is better in the F-GNR is likely because the reference configuration is closer to the crossover point - the increase in $\theta$ from the reference configuration to the crossover point is $\approx 0.03 \AA^{-1}$ for the F-GNR as opposed to $\approx 0.06 \AA^{-1}$ for the H-GNR. This results in less inaccuracy from the inextensibility approximation. For example, the twist-shortening effect would decrease the radius and increase the curvature and therefore the bending strain energy relative to the inextensible model. This effect is lessened when the reference configuration is closer to the crossover.

Energetically, the rod model behaves in a very simple way - when in a helical state, $W_{\tau}$, the torsional component of the energy remains constant at the same value that occurs in pure torsion at the crossover $\theta$. All additional twist is absorbed into the bending strain energy. This observation is important because it predicts that the stress-free state will never be helical - unless acted on externally, a helical GNR can always release strain by decreasing its curvature to zero while keeping the torsion constant until the crossover point is reached. Torsion will then be further reduced until the optimal (finite or zero) twist is reached in a state of pure torsion, or a completely straight state. These energetics are a result of the assumption that the bending component of the strain energy is of the form in Eq. (5). The good agreement between the rod model and the simulation results confirms the accuracy of this assumption and represents an insight into the behavior of FGNR edges. The torsion causes the edge $\mathrm{F}$ atoms to remain staggered, which precludes intrinsic bending, which, in turn, precludes the formation of an equilibrium helix configuration.

Here, the formation of a helix is studied by applying torsion to the GNR, essentially in the form of displacement boundary conditions. In experiment, helical GNRs have been observed confined within a CNT. ${ }^{1,3}$ Because our analysis indicates that stress-free GNRs do not form helices regardless of their edge decoration, the van der Waals adhesion of the GNR to the CNT and the 
corrugation of the graphene-graphene sliding energy exert sufficient additional force and torque to keep the GNR in a helical configuration.

It should be noted that the analysis herein is peculiar to narrow GNRs. The rod model implicitly contains the assumption of a non-deformable cross-section, which is satisfactory here. However, for wider GNRs, cross-sectional warping is known to occur ${ }^{8}$ and to affect the properties of a helix formed under torsion, requiring consideration of a deformable cross-section if elasticity modeling is to be carried out. ${ }^{15}$ These models are full plate models including extensibility and out-of-plane bending, and require numerical solutions of the elasticity equations. If an even wider GNR is constrained to form a helix with a large radius, such as by being confined within a CNT or wrapped around one, it can be reasonably modeled ${ }^{14}$ to be in a state of pure bending only. The reason why this analysis holds for a wide GNR can be understood as follows. The torsional stiffness scales as the square of the GNR width, ${ }^{16}$ while the bending stiffness scales linearly with width. Thus, very wide GNRs are very stiff in torsion compared to bending. They would have their pure torsionhelical crossovers at relatively low twist rates, keeping the torsional strain at a small value while increasing the bending significantly as the twist rate increases.

In conclusion, using objective boundary conditions in combination with SCC-DFTB, we modeled narrow (six dimers in width) GNRs with armchair edges terminated with F and H. F-GNRs have pure intrinsic twist with $\mathrm{F}$ atoms in a staggered configuration but intrinsic bending when the $\mathrm{F}$ atoms are on a same-side configuration. The H-GNRs have neither intrinsic twist nor bending. We observed the transition between a state of pure torsion and a helical configuration, caused by the relatively low energetic cost of taking on bending strain compared to increasing the torsional strain above a certain twist rate. We demonstrate that if the pure bending and pure torsion behavior of these GNRs is known, a simple rod model can predict the transition point, the energetics, and the morphologies of the helical configurations. The rod model shows that the stress-free configuration is always pure torsion or free of torsion, and external force is required to form a helix. In F-GNR, when twist rate is increased beyond its equilibrium value, a helical configuration with partial bending strain develops. However, the $\mathrm{F}$ atoms remain staggered and intrinsic bending does not arise. 
Thus, neither the simulations nor the rod model predict the existence of a helical equilibrium. Nevertheless, the edge chemistry plays an important role. Because of the propensity towards intrinsic twist in the F-GNR, it transitions to a helical configuration at a larger twist rate than the H-GNR. The F-GNR tends to form tighter (smaller radius) helices even at higher twist rates. Thus, GNRs with intrinsic twist are more prone to helical packing into CNTs with narrow diameters.

\section{Acknowledgement}

Work supported by NSF Grant No. CMMI-1332228. Computations were carried out at the Minnesota Supercomputing Institute.

\section{References}

(1) Chuvilin, A.; Bichoutskaia, E.; Gimenez-Lopez, M. C.; Chamberlain, T. W.; Rance, G. A.; Kuganathan, N.; Biskupek, J.; Kaiser, U.; Khlobystov, A. N. Self-Assembly of a SulphurTerminated Graphene Nanoribbon within a Single-Walled Carbon Nanotube. Nature Mater. 2011, 10, 687-692.

(2) Talyzin, A. V.; Anoshkin, I. V.; Krasheninnikov, A. V.; Nieminen, R. M.; Nasibulin, A. G.; Jiang, H.; Kaupinnen, E. I. Synthesis of Graphene Nanoribbons Encapsulated in SingleWalled Carbon Nanotubes. Nano Lett. 2011, 11, 4352-4356.

(3) Chamberlain, T. W.; Biskupek, J.; Rance, G. A.; Rance, G. A.; Chuvilin, A.; Alexander, T. J.; Bichoutskaia, E.; Kaiser, U.; Khlobystov, A. N. Size, Structure, and Helical Twist of Graphene Nanoribbons Controlled by Confinement in Carbon Nanotubes. ACS Nano 2012, 6, 3943-3953.

(4) Son, Y.W.; Cohen, M.L.; Louie, S.G. Energy Gaps in Graphene Nanoribbons. Phys. Rev. Lett. 2006, 97, 216803. 
(5) Hod, O.; Barone, V.; Peralta, J. E.; Scuseria, G. E. Enhanced Half-Metallicity in EdgeOxidized Zigzag Graphene Nanoribbons. Nano Lett. 2007, 7, 2295-2299.

(6) Hod, O; Scuseria, G.E. Electromechanical Properties of Suspended Graphene Nanoribbons. Nano Lett. 2009, 9, 2619-2622.

(7) Gunlycke, D.; Li, J.; Mintmire, J.W.; White, C.T. Edges Bring New Dimension to Graphene Nanoribbons. Nano Lett., 2010, 10, 3638-3642.

(8) Zhang, D.-B.; Dumitrică, T. Effective-Tensional-Strain-Driven Bandgap Modulations in Helical Graphene Nanoribbons. Small 2011, 7, 1023-1027.

(9) Zhang, D.-B.; Dumitrică, T. Role of Effective Tensile Strain in Electromechanical Response of Helical Graphene Nanoribbons with Open and Closed Armchair Edges. Phys. Rev. B 2012, 85,035445 .

(10) Lebedeva, I.V.; Popov, A.M.; Knizhnik, A.A.; Khlobystov, A.N.; Potapkin, B.V. Chiral Graphene Nanoribbon Inside Carbon Nanotube: Ab Initio Study. Nanoscale 2012, 4, 45224529.

(11) Kou, L.; Tang, C.; Wehling, T.; Frauenheim, Th.; Chen, C. Emergent Properties and Trends of a New Class of Carbon Nanocomposites: Graphene Nanoribbons Encapsulated in a Carbon Nanotube. Nanoscale 2013, 5, 3306-3314.

(12) Perim, E.; Fonseca, A.F.; Pugno, N.M.; Galvao, D. S. Violation of the Universal Behavior of Membranes inside Cylindrical Tubes at Nanoscale. EPL 2014, 105, 56002.

(13) Jiang, Y.; Li, H.; Li, Y.; Yu, H.; Liew, K. M.; He, Y.; Liu, X. Helical Encapsulation of Graphene Nanoribbon into Carbon Nanotube. ACS Nano 2011, 5, 2126-2133.

(14) Padra, N.; Song, Y.; Král, P. Self-assembly of Graphene Nanostructures on Nanotubes. ACS Nano 2011, 5, 1798-1804. 
(15) Cranford, S.; Buehler, M. J. Twisted and Coiled Ultralong Multilayer Graphene Ribbons. Modelling Simul. Mater. Sci. 2011, 19, 054003.

(16) Akatyeva, E.; Dumitrică, T. Chiral Graphene Nanoribbons: Objective Molecular Dynamics Simulations and Phase-Transition Modeling. J. Chem. Phys. 2012, 137, 234702.

(17) Dumitrică, T.; James, R.D. Objective Molecular Dynamics. J. Mech. Phys. Solids 2007, 55, 2206-2236.

(18) Elstner, M.; Porezag, D.; Jungnickel, G.; Elsner, J.; Haugk, M.; Frauenheim, Th.; Suhai, S.; Seifert, G. Self-Consistent-Charge Density-Functional Tight-Binding Method for Simulations of Complex Materials Properties. Phys. Rev. B 1998, 58, 7260.

(19) Aradi, B.; Hourahine, B.; Frauenheim, Th. DFTB+, A Sparse Matrix-based Implementation of the DFTB Method. J. Phys. Chem. A 2007, 111, 5678-5684.

(20) Köhler, C.; Frauenheim, Th. Molecular Dynamics Simulations of CFx (x=2,3) Molecules at $\mathrm{Si}_{3} \mathrm{~N}_{4}$ and $\mathrm{SiO}_{2}$ Surfaces. Surf. Sci. 2006, 600, 453-460.

(21) James, R.D. Objective Structures. J. Mech. Phys. Solids 2006, 54, 2354-2390.

(22) Zhang, D.-B.; Hua, M.; Dumitrică, T. Stability of Polycrystalline and Wurtzite Si Nanowires via Symmetry-Adapted Tight-Binding Objective Molecular Dynamics. J. Chem. Phys. 2008, 128, 084104.

(23) Zhang, D.-B.; Dumitrică, T.; Seifert, G. Helical Nanotube Structures of $\mathrm{MoS}_{2}$ with Intrinsic Twisting: an Objective Molecular Dynamics Study. Phys. Rev. Lett. 2010, 104, 065502.

(24) Zhang, D.-B.; James, R.D.; Dumitrică, T. Dislocation Onset and Nearly Axial Glide in Carbon Nanotubes under Torsion. J. Chem. Phys. 2009, 130, 071101.

(25) Zhang, D.-B.; Akatyeva, E.; Dumitrică, T. Bending Ultrathin Graphene at the Margins of Continuum Mechanics. Phys. Rev. Lett. 2011, 106, 255503. 
(26) Nikiforov, I.; Dontsova, E.; James, R.D.; Dumitrică, T. Tight-Binding Theory of Graphene Bending. Phys. Rev. B 2014, 89, 155437.

(27) Dontsova, E.; Dumitrică, T. Nanomechanics of Twisted Mono- and Few-layer Graphene Nanoribbons. J. Phys. Chem. Lett. 2013, 4, 2010-2014.

(28) Nikiforov, I.; Hourahine, B.; Aradi, B.; Frauenheim, Th.; Dumitrică, T. Ewald Summation on a Helix: A Route to Self-consistent Charge Density-Functional Based Tight-Binding Objective Molecular Dynamics. J. Chem. Phys. 2013, 139, 094110.

(29) Kirchhoff, G. Über dat Gleichgewicht und die Bewegung eines Unendlich Dünnen Elsastichen Stabes. J. Reine Angew. Math. (Crelle) 1859, 56, 285-313.

(30) Chouaeib, N.; Goriely, A.; Maddocks, J.H. Helices. Proc. Nat. Acad. Sci. U.S.A 2006, 103, 9398-9403.

(31) Chouaeib, N.; Maddocks, J.H. Kirchhoff's Problem of Helical Equilibria of Uniform Rods. J. Elast. 2004, 77, 221-247.

(32) Elstner, M. The SCC-DFTB Method and its Application to Biological Systems. Theor. Chem. Assoc. 2006, 116, 316-325.

(33) Singh, S.K.; Srinivasan, S.G.; Neek-Amal, M.; Costamagna, S.; van Duin, A.C.T.; Peeters, F.M. Thermal Properties of Fluorinated Graphene. Phys. Rev. B 2013, 87, 104114. 